\title{
Application of Geometric Polarization to Invariance Properties in Bistatic Scattering
}

\author{
D. H. O. Bebbington ${ }^{1}$, L. Carrea ${ }^{1}$, and G. Wanielik ${ }^{2}$ \\ ${ }^{1}$ Dept. of ESE, University of Essex, Wivenhoe Park, Colchester CO4 3SQ, UK \\ ${ }^{2}$ Professur Nachrichtentechnik, Chemnitz University of Technology, Reichenhainer Str. 70, 01126 Chemnitz, Germany
}

\begin{abstract}
Bistatic polarimetric radars provide target properties which just one monostatic system can not reveal. Moreover, augmentation of monostatic systems through the provision of bistatic receive-only stations can be a cheap way to increase the amount of remote sensing data. However, bistatic scattering needs to be investigated in order to properly define target properties such as symmetries and invariance, especially regarding choices of polarization basis. In this paper we discuss how the geometric theory of polarization, in which the geometry of the Poincaré sphere is directly related to 3-D geometry of space rather than the 2-D geometry of the wavefront plane, can be used to reduce the ambiguities in the interpretation of data. We also show how in the coherent case a complex scalar invariant can be determined irrespective of the basis combinations.
\end{abstract}

\section{Introduction}

Bistatic polarimetric radars may potentially increase the available density of remote sensing radar data significantly, as relatively cheap secondary receive-only systems, operating bistatically against a single transmitter can supplement a conventional monostatic system. Some bistatic geometries for remote sensing have been already proposed, such as the Cartwheel and pendulum configurations, for SAR interferometric measurements (Krieger et al., 2003). Moreover, bistatic phenomena such as the Brewster effect can reveal target properties that are not really manifest in monostatic scattering. Nevertheless, the understanding of general target properties - such as symmetries and invariance - in the bistatic case is less advanced than is the case for monostatic radar technique. This broader problem is further complicated by the fact that there are extra degrees of freedom in the choices of polarization basis, so that equivalent measurements are less readily compared in terms of the matrix properties alone. The outline of the problem to be addressed is that for bistatic scattering there is an equivalence relation,

$\mathbf{S} \sim \mathbf{U}_{r} \mathbf{S}_{0} \mathbf{U}_{t}^{T}$,

Correspondence to: L. Carrea

(lcarrea@essex.ac.uk) where $\mathbf{U}_{r}$ and $\mathbf{U}_{t}$ are unitary matrices representing polarization basis transformations at the receiving and the transmitting antenna, respectively. Since the group $U(2)$ has four parameters (Murnaghan, 1962), and each of the unitary matrices can be chosen independently, it is very difficult to understand which properties of the scattering matrix are due to the scatterer and which are due to the choice of the basis. Geometry is the right way since it establishes relations independently on coordinate systems. Furthermore, some formal questions have tormented polarimetric people for a long time, e.g. the incompatibility of Hermitian product for Jones vectors (Wanielik, 1988) or in general for complex spinors and Euclidean product for vectors in $\mathbb{R}^{2}$ or the choice of the group theory, $S U$ (2) for change of polarization basis and $S O(3)$ for rotations.

Geometry offers a single descriptive framework for defining states of polarization for different wave states and provides answers to the formal problems. Since Maxwell's equations can be reduced to differential geometry (Misner et al., 1973), it seems very natural to define the state of polarization using geometry directly from the electromagnetic field. Operatively, the use of geometry is translated into the employment of spinors and their projective "partners". They provide a valuable mathematical language which allows to:

- deduce the state of polarization in any frame directly from the full electromagnetic tensor,

- describe the field with the familiar Jones vector (Jones, 1947) via a reference spinor,

- express scattering matrices within a geometric framework,

- find geometric properties which can be interpreted as invariant classification for bistatic scattering,

- express directly in space-time the complex objects representing polarization,

- relate the Poincaré sphere directly to the 3-D geometry of the space. 


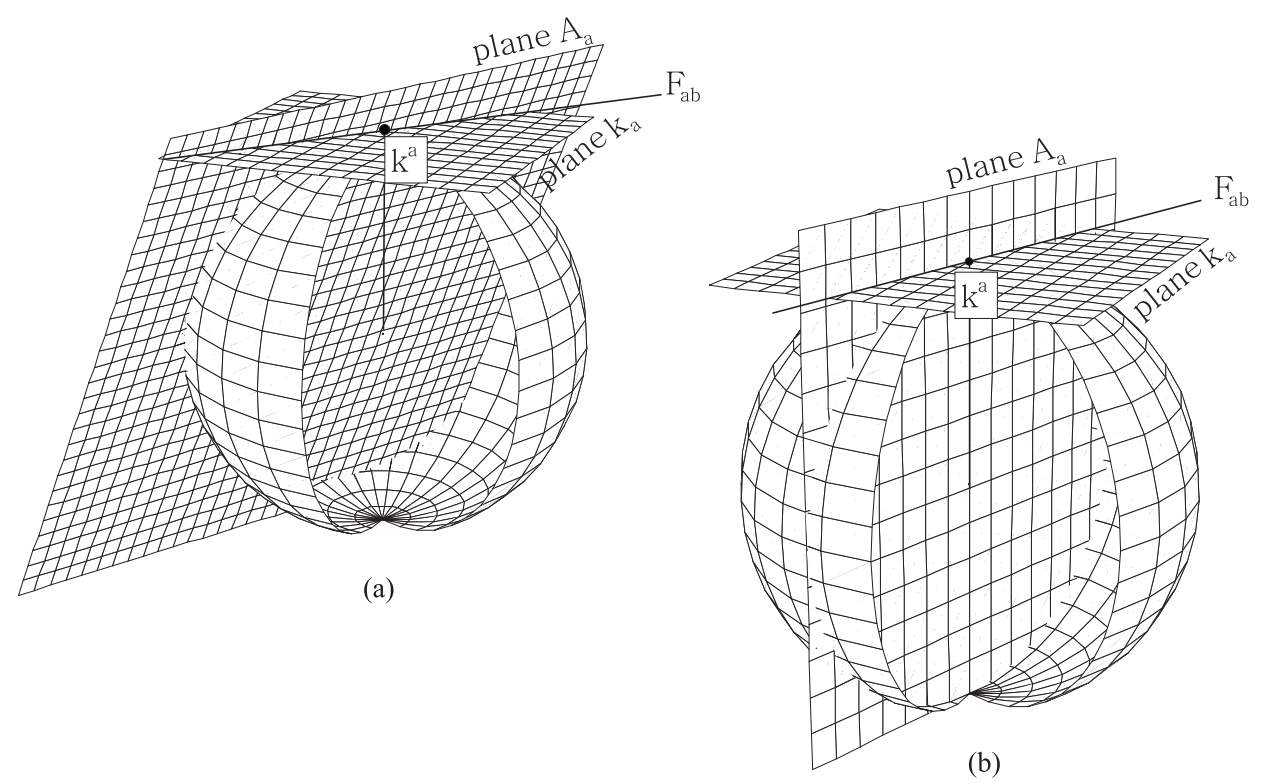

Fig. 1. Geometric relation between waveplane, vector potential and the electromagnetic field. The left side (a) shows one of the possible plane $A_{a}$ whose intersection is the same $F_{a b}$ line. The right side (b) shows the plane $A_{a}$ for the Coulomb gauge, the one through the origin.

\section{Projective representation of polarization states}

Polarization states can be defined directly from the electromagnetic field for harmonic waves which can be described in terms of the 4-vector potential (Post, 1997) as

$F_{a b}=i\left(k_{a} A_{b}-k_{b} A_{a}\right)$,

where $F_{a b}$ is the skew-symmetric field tensor, $A_{a}$ the 4 -vector potential and $k_{a}$ is the wave 4-vector with spacetime components:

$k_{a}=\left(\frac{\omega}{c}, k_{1}, k_{2}, k_{3}\right)$,

where $\omega$ is the angular frequency, $c$ the light speed, and $\left(k_{1}, k_{2}, k_{3}\right)$ the components of the conventional wave 3 -vector $\boldsymbol{k}$. The definition of the polarization state is embedded in Eq. (2) and it comes out from the projective interpretation of this relation. If the four coordinates of the wave covariant 4-vector $k_{a}$ (Rindler, 1991) are considered not as space-time coordinates but as homogeneous projective coordinates (Semple and Kneebone, 1952), the wave 4-vector can be visualized as a tangent plane to the wave sphere:

$k^{a} k_{a}=0 \Rightarrow\left[\begin{array}{l}k^{0} \\ k^{1} \\ k^{2} \\ k^{3}\end{array}\right]^{T}\left[\begin{array}{cccc}1 & 0 & 0 & 0 \\ 0 & -1 & 0 & 0 \\ 0 & 0 & -1 & 0 \\ 0 & 0 & 0 & -1\end{array}\right]\left[\begin{array}{l}k^{0} \\ k^{1} \\ k^{2} \\ k^{3}\end{array}\right]=0$,

where the superscript $T$ denotes the transpose operation. This relation expresses that the wave 4-vector is null and it can be interpreted as the homogeneous equation for $k_{a}$. Similarly, the 4-vector potential is a covariant vector, and the relation (2) states that the electromagnetic field tensor $F_{a b}$ is the intersection of the plane $k_{a}$ and the plane $A_{a}$ that is a line, as shown in Fig. 1.
Since for harmonic waves, the Maxwell equations imply

$k^{a} F_{a b}=0$,

the line $F_{a b}$ goes through the point $k^{a}$, polar (contravariant version of the wave 4-vector) to the plane $k_{a}$ (Semple and Kneebone, 1952). From Fig. 1a we can notice that the same $F_{a b}$ results from any $A_{a}$ that has the $k_{a}$ component added, hinging around $F_{a b}$. This is the geometric manifestation of the gauge freedom (Landau and Lifshitz, 1995). For harmonic fields, the appropriate choice for this degree of freedom is the so-called Coulomb gauge. It can be expressed as:

$\omega_{a} A^{a} \equiv\left[\begin{array}{l}1 \\ 0 \\ 0 \\ 0\end{array}\right]\left[\begin{array}{c}0 \\ A^{1} \\ A^{2} \\ A^{3}\end{array}\right]=0=\omega^{a} A_{a}$.

This relation can be geometrically interpreted with the following statements:

- the point $A^{a}$ belongs to the plane $\omega_{a}$,

- the plane $A_{a}$ goes through the origin of the sphere $\omega^{a}$ (the plane shows in Fig. 1b),

since any covariant vector, like $A_{a}$ is represented by a plane and any contravariant vector like $A^{a}$ by a point being polar to the plane with respect to the sphere (Semple and Kneebone, 1952). We are now ready to define the polarization state for any wave state using this plane at infinity, the polar to the origin of the sphere. In this plane, $k^{a}$ is projected as a line of intersection with $k_{a}$ a plane tangent to the sphere. Fixing one wave vector, the potential is also fixed as a point on this line belonging to the plane at infinity as shown in Fig. 2. Since the 


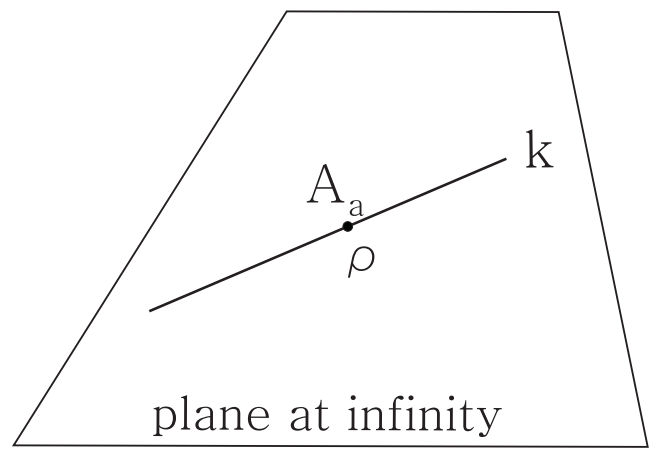

Fig. 2. The definition of the polarization for all possible wave directions as a point $A^{a}$ on the line $k^{a}$ on the plane at infinity.

gauge has been fixed, the only information contained in the potential is the polarization of the wave which is defined as a complex point $\rho$ on a line describing a wave direction. On each line it is always possible to find reference points that build the polarization basis, finding the intersection of the line with the conic generated by the intersection of the plane $A_{0}=0$ with the wave sphere. Any other polarization can be obtained as linear combination of the reference points.

\section{The scattering in the Argand plane}

Once the polarization has been defined, the general bistatic scattering can be introduced on the plane at infinity as a 1:1 relation between points on two different lines as shown in Fig. 3.

In order to establish such relation, we can recall the Moebius mapping between non-singular $2 \times 2$ complex matrices and bilinear transformations (Ahlfors, 1985). The scattering matrix turns out to be proportional to a matrix whose elements are the coefficients of the transformation of polarization ratio, the complex number $\rho$ :

$\rho=\frac{\alpha \rho_{0}+\beta}{\gamma \rho_{0}+\delta}$,

that can be written as the homogeneous equation:

$\gamma \rho_{0} \rho-\alpha \rho_{0}+\delta \rho-\beta=0$.

This equation has a very important physical interpretation: in the bistatic case to every transmitting antenna state $\rho_{0}$ corresponds a received polarization $\rho$ for which the voltage is null. In this way, Eq. (7) defines a 1:1 relation between the points $\rho_{0}$ and $\rho$ on different lines in the plane.

\section{The bistatic scattering as a homography}

So as to define the bistatic scattering we have to find the geometric object representing Eq. (7) and then physically interpret its geometric properties. The Eqs. (7) or (8) describes a homography, a 1:1 relationship between points on two lines in the projective plane. Homographies have two important properties (Semple and Kneebone, 1952):

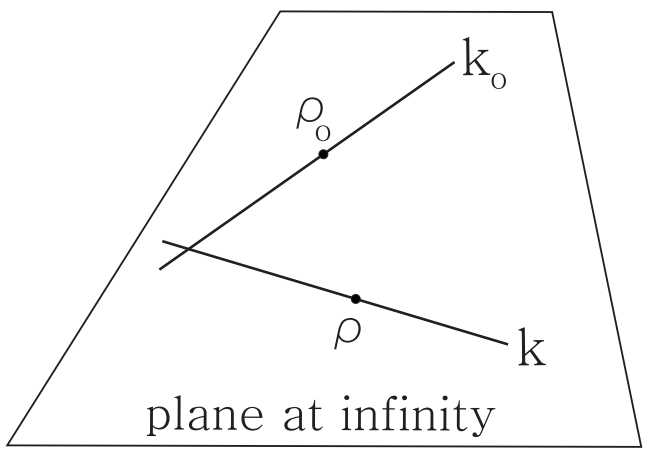

Fig. 3. Geometric representation of bistatic scattering.

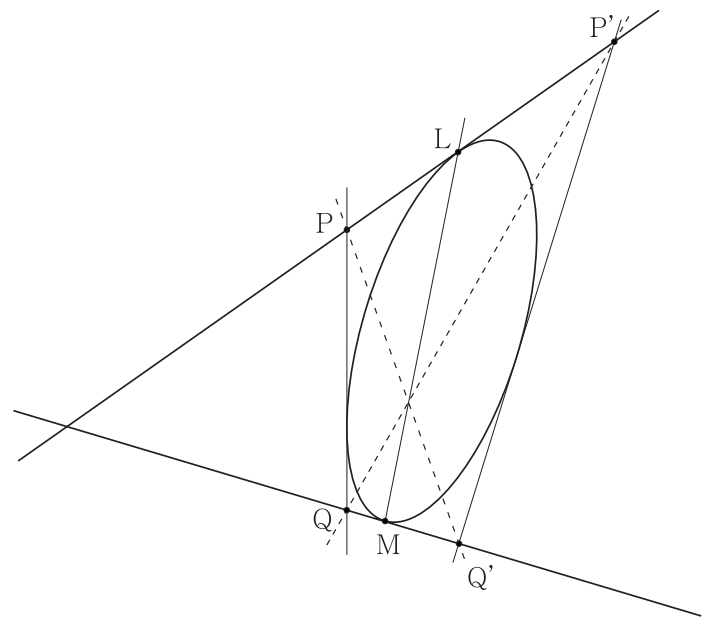

Fig. 4. The homographic correspondence between points of two lines.

- cross axis theorem: given a set of corresponding points $P Q, P^{\prime} Q^{\prime}$, etc., lines joining cross points $P Q^{\prime}$ and $P^{\prime} Q$ intersect on the axis $L M$ which passes through the points of the conic tangent to the two lines, as shown in Fig. 4,

- lines joining corresponding points between homographically related ranges envelop a conic. In this way, the conic can be used to obtain the corresponding points.

Now we are ready to physically interpret these geometric statements: for any pair of wave vectors $k_{0}$ and $k$, corresponding points $P$ and $Q$, solutions of Eq. (8), are the mutually nulled antenna polarization states $\rho_{0}$ (transmitting antenna state) and $\rho$ (receiving antenna state), such that the line joining them is tangential to a conic.

It is very important to notice that the relation found exists independent of any choice of coordinates, and we can assert that any invariant property of a homography is characteristic of the scattering matrix in the general bistatic case. 


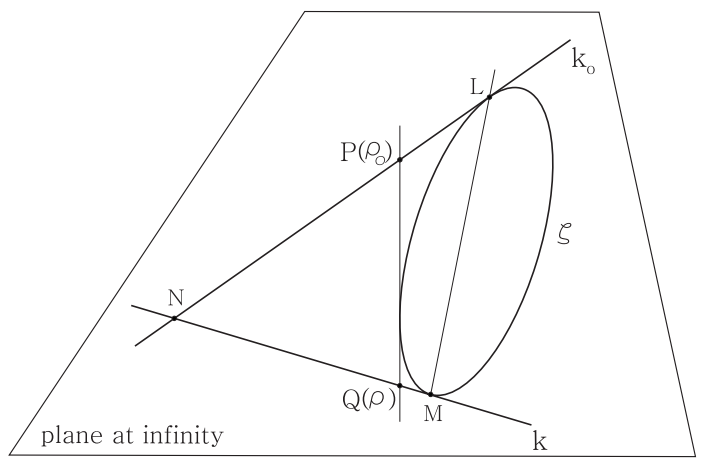

Fig. 5. Geometric representation of bistatic scattering.

\section{Canonical basis, canonical decomposition and the complex scalar invariant}

For the general bistatic case, we can define a triangle whose vertices are the special points $L$, the intersection of the two lines $k_{0}$ and $k$, tangent to the conic, $M$ and $N$ special points belonging to the conic, as shown in Fig. 5. We can express points on $k_{0}$ and $k$ (that is polarization states) using $N$ and $L$ for $k_{0}$ and $N$ and $M$ for $k$ as reference points. We can also define the scattering matrix, represented by the conic $S$ using this basis in a very simple form. However, it can be shown that this basis is not generally unitary. Using properties of homographies, a suitable change of basis can be applied to move from the basis related to the triangle of reference to pairs of unitarily orthogonal states. It is necessary to create for each wave vector a reflexive homography that maps the characteristic polarizations to pairs of orthogonal states, for example circular polarizations ${ }^{1}$. The resulting homography acting on the local basis turns out to be symmetric and produce symmetric matrices. The canonical decomposition of the natural basis bistatic scattering matrix can be summarized as:

$\mathbf{S}=\mathbf{S}_{r} \mathbf{S}_{0} \mathbf{S}_{t}$,

where $\mathbf{S}_{r}$ and $\mathbf{S}_{t}$ are symmetric scattering matrices and $\mathbf{S}_{0}$ is canonical:

$\mathbf{S}_{0}=\left[\begin{array}{cc}0 & \zeta \\ -1 & 0\end{array}\right]$

and the complex parameter $\zeta$ characterizing the matrix $\mathbf{S}_{0}$ can be measured. The matrices $\mathbf{S}_{r}$ and $\mathbf{S}_{t}$ are symmetric and they can be related to the characteristic polarizations. In order to construct them, we consider the physical meaning of $N, L$ and $M$ :

- $N$ is determined by the $k$ wave vectors: it represents the intersection between two wave planes, that is the normal to the scattering plane,

- $L$ and $M$ can be determined using the tangent property defined in Sect. 4. The tangent points $L$ and $M$ are those

\footnotetext{
${ }^{1}$ It can be shown that this choice is not arbitrary.
}

that correspond to $N$ : transmitting a linear polarization along $N$, the same antenna will receive a null voltage for the polarization $L$ (or $M$ ).

The characteristic polarizations are enough to build the symmetric scattering matrices $\mathbf{S}_{r}$ and $\mathbf{S}_{t}$. It is possible to show that the conic $\mathbf{S}_{0}$ is completely characterized by the complex scalar $\zeta$ and the theory of invariance of conics establishes that $\zeta$ is a projective invariant (Semple and Kneebone, 1952). $\zeta$ can be considered as a polarimetric invariant independently of unitary transformations of transmitting or receiving polarization basis for a specific scattering mechanism.

\section{Backscattering case}

For backscattering, $k_{0}$ and $k$ are coincident lines of opposite direction relating antennas pointing in the same direction. The conic $S_{0}$ degenerates becoming a pair of points which characterizes completely the scattering matrix. This pair of points is the pair of copolar nulls $\mu_{A}, v_{A}$ from which the scattering matrix can be built, up to a scale (Bebbington, 1997):

$S_{A B}=\frac{1}{2}\left(\mu_{A} v_{B}+v_{A} \mu_{B}\right)$.

In this way, the backscattering case can be easily represented in this geometric framework.

\section{Conclusions}

Geometric polarimetry represents an integrated approach to the theory of electromagnetic polarization. The use of geometry in polarimetry has been shown to be very effective for both formal questions and practical issues. The main result is that a complex scalar invariant $\zeta$ can be defined using the canonical form of the bistatic scattering matrix and measured, in addition to the extensive unitary invariants of a scattering matrix, such as power or energy.

Acknowledgements. This work has been supported by the European Research Training Network (RTN) AMPER, contract number HPRN-CT-2002-00205.

\section{References}

Ahlfors, L. V.: Clifford Numbers and Moebius Transformations in $\mathbb{R}^{n}$ in Clifford Algebras and Their Applications in Mathematical Physics, Proceedings of NATO and SERC Workshop, Canterbury, Kent, edited by: Chisholm and Common, NATO ASI Series (Reidel 1986), 1985.

Bebbington, D.: Analytical Foundations of Polarimetry: I, Application of Spinor Algebra as a Unified Approach to Coherent and Incoherent Polarimetry, WIPSS Expert Retreat, U.S. Office for Naval Research, DLR Institut für Hochfrequenztechnik, Oberpfaffenhofen, Germany, June 1997.

Jones, R. C.: A New Calculus for the Treatment of Optical Systems I: Description and Discussion, Journal Optical Society of America, vol. 37, 107-110, 1947. 
Krieger, G., Fiedler, H., Mittermayer, J., Papathanassiou, K., and Moreira, A.: Analysis of Multistatic Configurations for Spaceborne SAR Interferometry, IEE Proceedings Radar Sonar Navig., vol. 150, no.3, 87-96, 2003.

Landau, L. D. and Lifshitz, E. M.: The Classical Theory of Fields, 4th edition, Butterworth-Heinemann, 1995.

Misner, C. W., Thorne, K. S., and Wheeler, J. A.: Gravitation, W. H. Freeman and Company, 1973.

Murnaghan, F. D.: The Unitary and Rotation Groups, Spartan Books, Washington D.C., 1962.
Penrose, R. and Rindler, W.: Spinors and Space-Time, vol. 1, Cambridge University Press, 1984.

Post, E. J.: The Formal Structure of Electromagnetism, Dover Publications, 1997.

Rindler, W.: Introduction to Special Relativity, Clarendon Press, Oxford, 1991.

Semple, J. G. and Kneebone, G. T.: Algebraic Projective Geometry, Claredon Press, Oxford, 1952.

Wanielik, G.: Signaturuntersuchungen an einem polarimetrischen Pulsradar, Dissertation, Universität Karlsruhe Deutschland, VDI Verlag, 1988. 